\title{
A finite element method of modelling and designing of an ACCC conductor
}

\author{
Yan Youcan and Duan Yugang \\ State Key Lab for Manufacturing Systems Engineering, Xi'an Jiaotong University, Xi'an, China
}

\begin{abstract}
The ultimate breaking strength of conductors always plays a vital role in the long-term safe operation of transmission lines and it should be carefully considered in the designing of a conductor. While limited literature has been released regarding the strength calculation of ACCC (Aluminum Conductor Composite Core) conductors. In this study, a finite element method based on real conductor samples is proposed to evaluate the strength of the ACCC conductor, and investigate on how diameters (or sectional areas) of the composite core and aluminum strands influence the conductors' breaking strength, thereby helping engineers design a proper diameter of an ACCC conductor. The finite element analysis shows that increasing the sectional area of the composite core leads to a larger breaking strength than increasing an equal sectional areas of aluminum strands, which is in line with the tensile experimental results. In specific, the relative errors between the simulated breaking strength and the experimental results are as low as less than $3 \%$. Therefore, the finite element method presented in this work, to some extent, fills in the blank of strength calculation for ACCC conductors, and in practical terms, it could serves as guidelines for conductor-design engineers.
\end{abstract}

\section{Introduction}

In recent years, the Aluminum Conductor Composite Core (ACCC) conductor has been widely used in overhead transmission lines due to its merits of high specific strength, light weight, and low lag at high temperature compared with the traditional Aluminum Conductor Steel Reinforced (ACSR) conductor [1-5].

As shown in Figure 1, the ACCC conductor consists of two-layer aluminum strands and a composite core based on carbon fibers and glass fibers, which has successfully replaced the high weight steel core in ACSR conductors [5]. The inner strength core is based on continuous carbon fibers while the outer insulation layer is based on glass fiber/epoxy composites to prevent a direct electrical path between the aluminum conductors and the conductive carbon fibers. Both kinds of fibers are impregnated with epoxy and shaped into a composite mandrel bar [6]. Additionally, the out-layer aluminum strands of circular sections are replaced with strands of trapezoidal sections, which can increase the conductive areas, and at the same time, the two-layer aluminum strands are twisted in a reverse spiral to improve the stability of the wire structures. In this paper, the lay ratio (the ratio of screw pitch to the outer diameter) of the ACCC conductor samples is a constant.

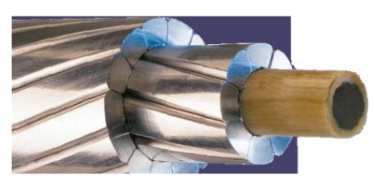

Figure 1. Macro-structure of an ACCC wire
It is well known that the ultimate tensile strength of conductors plays a vital role in the long-term safe operation of transmission lines, and it is important to take the breaking strength into consideration when designing a conductor. Prior to this study, plenty of work has been done both analytically and numerically to study the strength calculation methods for the ACSR conductor with circular cross sections [7-14]. Cardou A. et al. [12] developed concise mechanical models of ACSR helical strands, while it cannot be applied to analyze ACCC conductors with more complex sections - trapezoid sections (outer aluminum strands) plus a circular section (composite core). W.G. Jiang et al. [13] presented a theoretical finite element model for simple straight wire rope strand, and MA Jun et al. [14] also established a finite element model using ANSYS to simulate the stress and deformation of ACSR conductors, but limited literature has been released regarding the strength calculation of the ACCC conductor.

In this paper, a finite element method based on real conductor samples is proposed to evaluate the breaking strength of the ACCC conductor, and study how diameters (or cross-section areas) of the composite core and aluminum strands influence the conductors' breaking strength. Therefore, this method can promisingly help engineers design a new type of ACCC conductor, and to some extent, it fills in the blank of strength calculation for ACCC conductors.

\section{Method and Assumptions}

Prior to modelling, it's necessary to make some assumptions on materials of models so as to simplify the analysis. Firstly, the carbon composite core is regarded as ideal elastic body in axial direction on the premise of a 
small deformation, and only axial modulus and Poisson's ratio are required to be defined. In addition, as the aluminum strands in out layers have a relatively low axial modulus (around $60 \mathrm{GPa}$ compared to $120 \mathrm{GPa}$ of the composite core) and a high elongation percentage (more than $20 \%$ ), we assumed aluminum as bilinear elasticplastic body, which means that the nonlinear plastic deformation of the aluminum wire after reaching its yield limit $\sigma 0$ (about $80 \mathrm{MPa}$ ) in the elastomer stage is simplified as a linear deformation with a tiny modulus, i.e. Tangent Modulus G0 (around 0.6GPa).

Then the geometrical model of an ACCC conductor is created in ANSYS software based on real wire samples until it is meshed with solid185 eight-node elements. To match the practical situation, contact behaviors between conductor layers are taken into consideration and the flexible three-dimensional contact pairs are applied, in which the target surface adopts TARGE170 units and the contact surface adopts CONTA174 units. After completely constraining one end of the conductor and applying an axial displacement load to the other end, the model can be solved and the calculation results of the stress and deformation are obtained. By integrating the stress results on the cross section of the conductor, we can obtain the corresponding internal force, which is equivalent to the tensile load applied on the end of the wire.

Assuming that if the von Mises stress to any of the components in the ACCC wire material, i.e., the composite core or the aluminum strands reaches or exceeds its ultimate tensile strength, the wire would be considered as been broken (referring to the fourth strength theory or distortion energy theory in material mechanics). Therefore, we can obtain the breaking strength by increasing the axial displacement load stepwise until the maximum von Mises stress to the aluminum or composite core reaches its ultimate tensile strength.

Comparing the simulated breaking strength with the experimental results of tensile tests, we can verify the rationality of this finite element method, and figure out how diameters or cross-section areas of the composite core and aluminum strands influence the ultimate breaking strength of ACCC conductors. This can help engineers choose an optimal diameter of the composite and aluminum strands of ACCC wires on the ground that other mechanical and electrical requirements are satisfied.

\section{Finite Element Model}

\subsection{Geometric Modelling}

The finite element model is created on 1:1 based on, for example, the JRLX/T (ACCC) wire samples produced by Far East Composite Technology Co., Ltd., and the wire code in USA is Linnet ACCC/TW431 [15]. In this model, the rod length is set to $43.2 \mathrm{~mm}$ which approximates to a quarter of the wire pitch, the outer diameter of the wire is $18.29 \mathrm{~mm}$, and the diameter of the inner composite core is $5.97 \mathrm{~mm}$. Specific geometrical parameters of the JRLX/T wire are listed in Table 1 [16], and the specific mechanical properties of the JRLX/T wire are shown in Table 2.
Table 1. Geometrical parameters of the JRLX/T wire

\begin{tabular}{|c|c|}
\hline Parameters & Value \\
\hline Nominal total transverse area, $\mathrm{mm}^{2}$ & 246.4 \\
\hline Nominal aluminum transverse area, $\mathrm{mm}^{2}$ & 218.4 \\
\hline Outside diameter of aluminum wires, $\mathrm{mm}$ & 18.29 \\
\hline Diameter of composite core, $\mathrm{mm}$ & 5.97 \\
\hline Lay ratio of inner-layer aluminum strands & 19 \\
\hline Lay ratio of outer-layer aluminum strands & 10 \\
\hline Filling coefficient & 0.93 \\
\hline
\end{tabular}

Table 2. Mechanical properties of the JRLX/T wire

\begin{tabular}{|c|c|c|}
\hline Properties & $\begin{array}{c}\text { Composite } \\
\text { Core }\end{array}$ & $\begin{array}{c}\text { Aluminum } \\
\text { strands }\end{array}$ \\
\hline Axial Modulus $(\mathrm{GPa})$ & 120 & 60 \\
\hline Poisson's Ratio $\delta$ & 0.27 & 0.3 \\
\hline $\begin{array}{c}\text { Tangent Modulus } G_{0} \\
(\mathrm{GPa})\end{array}$ & $/$ & 0.6 \\
\hline Yield Limit $\sigma_{0}(\mathrm{MPa})$ & $/$ & 80 \\
\hline $\begin{array}{c}\text { Ultimate Tensile Strength } \\
\sigma_{m}(\mathrm{MPa})\end{array}$ & $\geq 2100$ & 90 \\
\hline
\end{tabular}

In general, the geometrical modelling process of the JRLX/T wire is based on a bottom-top method, which mainly includes four steps as follows:

1. Create the outline and bottom areas by connecting key points and boundary lines respectively (Figure 2a).

2. Create the sub-volume of an aluminum strand by copying, rotating the bottom area and connecting corresponding key points (Figure 2b).

3. Create the circular carbon core by extrusion and single aluminum strands by a similar way in step 2 (Figure 2c).

4. Complete the whole model by copying circumferentially (Figure 2d).

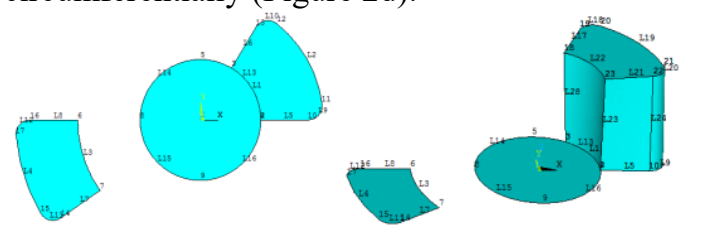

(a)

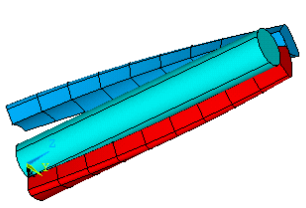

(c) (b)

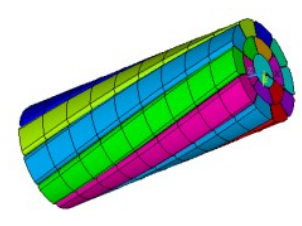

(d)
Figure 2. Procedure of creating a finite element model of the JRLX/T wire: (a) creation of the outline and bottom areas, (b) creation of the sub-volume of an aluminum strand, (c) creation of the circular carbon core and single aluminum strands, (d) creation of the complete wire model

\subsection{Contact Pairs Setting}

After meshing the finite element model (20,400 8-noded elements are generated), we set contact pairs between the composite mandrel and the inner aluminum strands, as 
well as contact pairs between the inner and outer layer of aluminum strands [16], which is well matched with the true conditions. As the ACCC rod are meshed with threedimensional solid185 elements, the types of contact elements are set as flexible three-dimensional TARGE170 and CONTA174. Using the contact wizard in ANSYS and displaying the contact pairs graphically, the contact pairs are shown in Figure 3a and 3b, respectively.

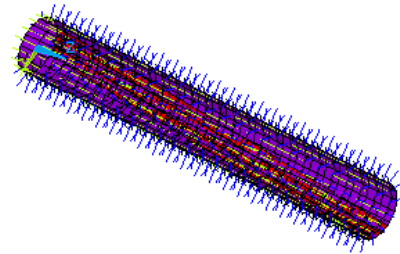

(a)

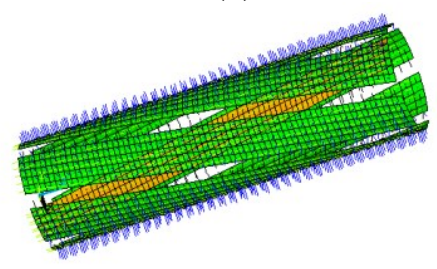

(b)

Figure 3. (a) Contact pair between the composite mandrel and inner aluminum strands, (b) Contact pair between the inner and outer layer of aluminum strands.

\subsection{Boundary Conditions}

Constraining all displacements (X, Y and $\mathrm{Z}$ directions) of nodes on the one end (bottom areas) of the wire model and only the radial displacements (X direction in cylindrical coordinates) of nodes on the other end (top areas), then applying axial displacement ( $\mathrm{Z}$ direction) loads $\Delta l$ on the top areas.

\subsection{Results and Discussions}

Increasing the axial displacement load $\Delta l$ stepwise from 0.05 to $1 \mathrm{~mm}$ and setting the number of sub-steps properly, we can obtain the desired stress results of the ACCC wire when the maximum von Mises stress to the aluminum or composite core reaches its ultimate tensile strength.

Figure 4 a give the curve of von Mises stresses to the nodes of the wire model over the axial displacement loads up to $0.75 \mathrm{~mm}$ within 24 sub-steps. To see more clearly, the curves of von Mises stresses to aluminum strands, namely SEQV-2 and SEQV-3 are independently shown in Figure $4 \mathrm{~b}$. Figure 5 shows the distribution of axial stresses on the mid-section of the ACCC wire. At the same time, the von Mises stresses to the nodes in Figure 4 are extracted and listed in Table 3 (at Time $=1$, i.e., $\Delta l=0.75 \mathrm{~mm}$ ).

The von Mises stress to the composite core (SEQV-1) in Figure 4a saw a constant increase and reaches up to $2127.19 \mathrm{MPa}$ when $\Delta l=0.75 \mathrm{~mm}$, which exceeded its ultimate strength limit $-2100 \mathrm{MPa}$ (referring to Table 2), indicating that the composite core had broken at that moment. In the meantime, the maximum von Mises stress to the two-layer aluminum strands SEQV-2 and SEQV-3 reaches $88.81 \mathrm{MPa}$ and $80.48 \mathrm{MPa}$ respectively, which exceeded its yield strength-80MPa while remaining smaller than its ultimate strength limit- $90 \mathrm{MPa}$, that is to say, the aluminum strands were deforming plastically while had not yet been broken.

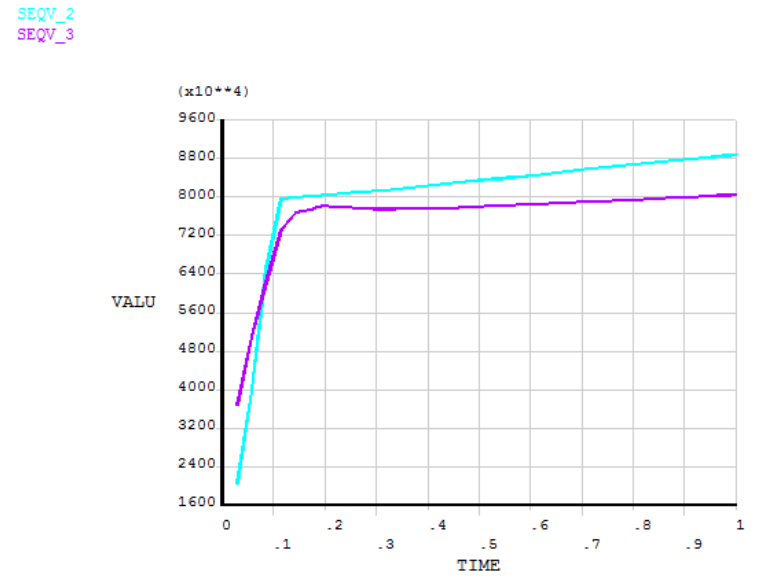

(a)

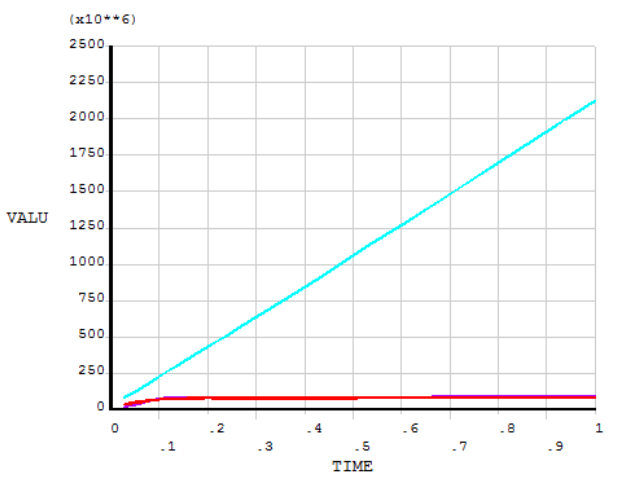

(b)

Figure 4. (a) Curve of von Mises stresses to the wire model over axial displacement loads, (b) Curve of von Mises stresses to the two-layer aluminum strands over axial displacement loads. (SEQV-1: the von Mises stress to the composite core, SEQV-2: the von Mises stress to the inner aluminum strands, SEQV-3: the von Mises stress to the outer aluminum strands)

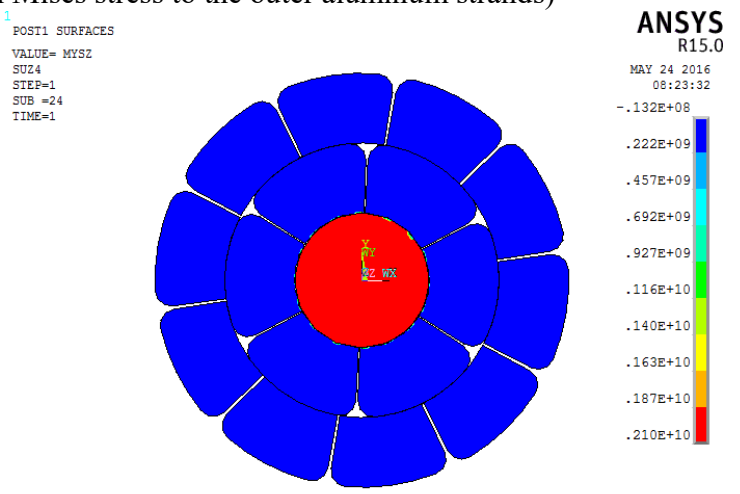

Figure 5. The distribution of axial stresses on the mid-section of the ACCC wire

Table 3. Maximum von Mises stresses to the nodes of components of the ACCC wire model

\begin{tabular}{|c|c|}
\hline Components of the ACCC Wire & $\begin{array}{c}\text { Maximum Von Mises } \\
\text { Stresses (MPa) }\end{array}$ \\
\hline Composite core & 2127.19 \\
\hline Inner-layer aluminum strands & 88.81 \\
\hline Outer-layer aluminum strands & 80.48 \\
\hline
\end{tabular}


Consequently, the equivalent axial force, i.e., the simulated tensile breaking force, can be calculated by integrating axial stresses on cross sections of the ACCC wire at last time-step (Time=1). At the same time, we can obtain the simulated breaking strength of ACCC conductors in different sizes using the same method, and the simulated results are listed in Table 4.

\section{Experiments}

The tensile testing experiments are carried out by Far East Composite Technology Co., Ltd. and guided by the Standards of Q/GDW 1851-2012, which is issued by State Grid Corporation of China. Firstly, the conductors sample are applied with special hardware at two ends so as to connect with the tensile testing machine. In the next step, the conductor is clamped by clamps on the tensile tester and the tensile load is applied on its one end at a loading speed of $20-40 \mathrm{kN} / \mathrm{min}$ [17]. The tests end when either the composite core or aluminum strands breaks. The experimental results of six conductor samples in various sizes are listed in Table 4.

As shown in Table 4, the simulated breaking load is highly consistent with the experimental results, with a low relative error of no more than $3 \%$, which implies that the finite element method in this work is able to predict the breaking load of an ACCC conductor reasonably in practice.

In addition, it is found in Figure 6 that the breaking strength of conductors increase as the sectional areas of the composite core and aluminum strands become larger, while the increase in sectional area of composite core contributes more to a greater breaking force than that of aluminum strands.

We can draw the conclusion aforementioned particularly by figures for Sample 4 and Sample 5-the experimental breaking strength $\left(F_{N 0}\right)$ increases by as large as $15.9 \mathrm{kN}$ (from 106.1 to 122.0 ) as the sectional area of the composite core $\left(\mathrm{S}_{\mathrm{C}}\right)$ rises by $7 \mathrm{~mm}^{2}$ and the sectional areas of aluminum strands $\left(\mathrm{S}_{\mathrm{A}}\right)$ increases by $10 \mathrm{~mm}^{2}$, while $F_{N 0}$ increases by only $2.9 \mathrm{kN}$ (from 103.0 to 106.1 ) and $2.2 \mathrm{kN}$ (from 122.0 to 124.2 ) as $\mathrm{S}_{\mathrm{A}}$ rises by $40 \mathrm{~mm}^{2}$ (from 310 to 350 ) and $20 \mathrm{~mm}^{2}$ (from 360 to 380 ) respectively, with $\mathrm{S}_{\mathrm{C}}$ stay constant in Sample 3,4 $\left(40 \mathrm{~mm}^{2}\right)$ and Sample $5,6\left(47 \mathrm{~mm}^{2}\right)$. It means that increasing the sectional area of the composite core can lead to a larger breaking strength than increasing an equal number of sectional areas of aluminum strands, which is in line with the real condition that the composite core bears most of the tensile load.

Table 4. Comparison between the simulated breaking s and the experimental one based on conductor samples of different sizes [15]

\begin{tabular}{|c|c|c|c|c|c|c|}
\hline Conductor Samples No. & 1 & 2 & 3 & 4 & 5 & 6 \\
\hline Sectional Area of the Composite Core $\mathrm{S}_{\mathrm{C}}\left(\mathrm{mm}^{2}\right)$ & 28 & 28 & 40 & 40 & 47 & 47 \\
\hline Sectional Area of Aluminum Strands $\mathrm{S}_{\mathrm{A}}\left(\mathrm{mm}^{2}\right)$ & 218 & 240 & 310 & 350 & 360 & 380 \\
\hline Simulated Breaking Strength $F_{N A}(\mathrm{kN})$ & 70.2 & 72.9 & 100.3 & 103.6 & 119.3 & 121.1 \\
\hline Experimental Breaking Strength $F_{N 0}(\mathrm{kN})$ & 72.1 & 74.8 & 103.0 & 106.1 & 122.0 & 124.2 \\
\hline Relative Error $\eta$ Between $F_{N A}$ and $F_{N 0}(\%)$ & 2.64 & 2.54 & 2.62 & 2.37 & 2.21 & 2.50 \\
\hline
\end{tabular}

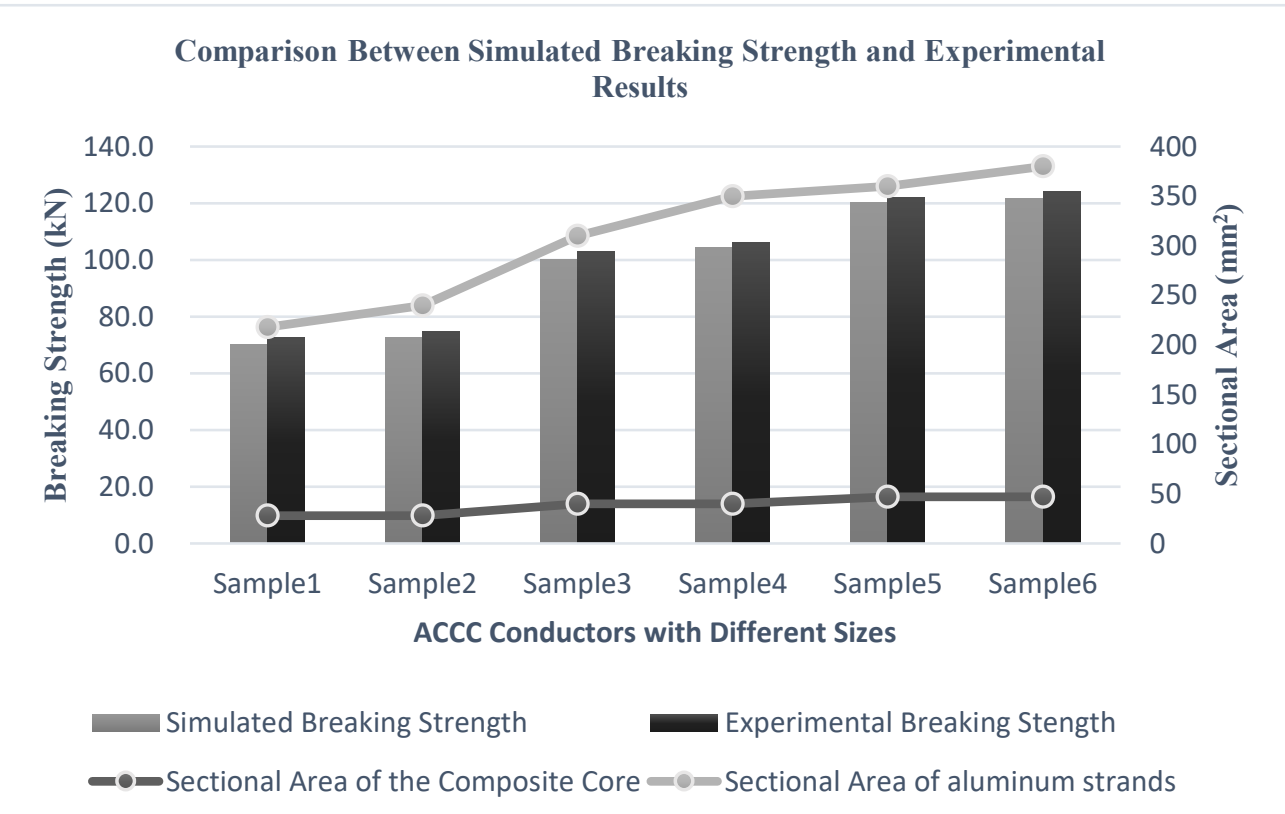

Figure 6. Comparison between simulated breaking strength and experimental results of conductor samples with different sizes

Overall, the increase in sectional area of composite core contributes more to a larger breaking force compared to that of aluminum strands. The calculated tensile breaking force $F_{N A}$ is very close to the experimental result 
$F_{N 0}$, and the relative error $\eta$ between simulations and experiments is less than 3\%, which indicates that the simulation result fits well with the actual breaking process.

\section{Conclusions}

Herein, a finite element method based on real ACCC conductor samples is proposed to investigate on how sectional areas of both the composite core and aluminum strands affects the breaking strength of ACCC conductors. The stress-displacement curves demonstrate that the composite core bears most of tensile load applied on the conductor, compared to the aluminum strands yielding first and deforming plastically afterwards (with no load-bearing capacity).

It is also found that a $7 \mathrm{~mm} 2$ increase in the sectional area of the composite core and a $10 \mathrm{~mm} 2$ increase in the sectional area of aluminum simultaneously lead to a rise by $15.9 \mathrm{kN}$ of breaking strength, while a $40 \mathrm{~mm} 2$ increase in the sectional area of aluminum solely produces a rise by only $2.9 \mathrm{kN}$ of breaking strength (keeping the sectional area of the composite core unchanged). It means that the breaking strength of conductors increase with a larger sectional area of both the composite core and aluminum strands, while the increase in the sectional area of composite core contributes more to a larger breaking force than that of aluminum strands. In addition, the calculated breaking force in conductors is highly consistent with the experimental results (with a low relative error of less than $3 \%$ ), which means that the finite element model fits well with the true condition.

The finite element method presented in this work, to some extent, fills in the blank of strength calculation for ACCC conductors, and in practical terms, it could serves as guidelines for conductor-design engineers. For example, they can choose an optimal diameter of the composite and aluminum strands of ACCC wires based on practical requirements (current-carrying capacity, weight, rated breaking strength, etc.), and for conductors operating in extreme conditions (heavy wind, ice or snow), a larger diameter of the composite core is highly recommended to meet the strength requirements.

However, this paper focuses on the strength evaluation of ACCC conductors in room temperature (around $20^{\circ} \mathrm{C}$ ), and in most cases, the mechanical performance of wires under high temperature (around $300^{\circ} \mathrm{C}$ ) should be considered. In future work, we will investigate on how temperature affects the breaking strength and the mechanical creep of ACCC conductors as the carbon composite core and aluminum strands have different expansion coefficients and so forth.

\section{References}

1. Alawar, E.J. Bosze, and S.R. Nutt, "A Composite Core Conductor for Low Sag at High Temperatures," IEEE Trans. Power Delivery, Vol. 20, pp. 2193-2199, (2005).

2. E.J. Bosze, A. Alawar, Y.I. Tsai, S.R. Nutt, D. Bryant, and G. Bowles,"Performance of a New Overhead
Conductor Design using a Carbon/Glass Fiber Composite Core", Intern. Conf. Overhead Lines, Fort Collins, Colorado, USA, pp. 121-135, (2006).

3. E.J. Bosze, Y.I. Tsai, E. Barjasteh, S.R. Nutt, and D. Bryant, "Long-term Durability of the Composite Core Conductor ACCC/TW', Intern. Conf. Overhead Lines, Fort Collins, Colorado, USA, pp. 516-525, (2008)

4. $3 \mathrm{M} \odot$ Company, "Aluminum Conductor Composite Reinforced Technical Notebook (477 kcmil family)," (C) (March 2003), v.2.21.

5. Berjozkina S, Sauhats A, Bargels $\mathrm{V}$, et al. The technical and economic efficiency of using conductors with composite core in the transmission grid $[\mathrm{C}] / /$ International Conference on the European Energy Market. IEEE, (2012):1-7.

6. Burks B, Armentrout D L, Kumosa M. Failure prediction analysis of an ACCC conductor subjected to thermal and mechanical stresses[J]. IEEE Transactions on Dielectrics and Electrical Insulation, (2010), 17(2).

7. Páczelt I, Beleznai R. Nonlinear contact-theory for analysis of wire rope strand using high-order approximation in the FEM[J]. Computers \& Structures, (2011), 89(11):1004-1025.

8. Stanova E, Fedorko G, Fabian M, et al. Computer modelling of wire strands and ropes Part I: Theory and computer implementation $[\mathrm{J}]$. Advances in Engineering Software, (2011), 42(6):305-315.

9. Frikha A, Cartraud P, Treyssède F. Mechanical modeling of helical structures accounting for translational invariance. Part 1: Static behavior[J]. International Journal of Solids \& Structures, (2013), 50(9):1373-1382.

10. Jiang W G, Henshall J L, Walton J M. A concise finite element model for three-layered straight wire rope strand $[\mathrm{J}]$. International Journal of Mechanical Sciences, (2000), 42(1):63-86.

11. Kumar K, Cochran J E. Closed-Form Analysis for Elastic Deformations of Multilayered Strands[J]. Journal of Applied Mechanics, (1987), 54(4):744-744.

12. Cardou A, Jolicoeur C. Mechanical Models of Helical Strands[J]. Applied Mechanics Reviews, (1997), 50(1): 1 .

13. Jiang W G, Yao M S, Walton J M. A concise finite element model for simple straight wire rope strand $[\mathrm{J}]$. International Journal of Mechanical Sciences, (1999), 41(2): 143-161.

14. Jun M A, Ge S R, Zhang D K. Distribution of wire deformation within strands of wire ropes $[\mathrm{J}]$. Journal of China University of Mining and Technology, (2008), 18(3): 475-478.

15. JRLX/T (ACCC) Wire products, Far East Holding Composite Technology Co., Ltd[z], (2009).

16. Gnanavel B K. Effect of interfacial contact forces in radial contact wire strand $[\mathrm{J}]$. Archive of Applied Mechanics, (2011), 81(3):303-317.

17. GB/T 1179-2008, Round wire concentric lay overhead electrical stranded conductors [S], (1991). 